\title{
Perminvar Effect in Amorphous Hitperm Alloy
}

\author{
M. Varga, R. Varga, P. Vojtanik \\ Institute of Physics, Fac. Sci., P.J. Šafárik University \\ Park Angelinum 9, 04154 Košice, Slovakia \\ AND E. KOMOVA \\ Faculty of Aeronautics, Technical University \\ Rampová 7, 04121 Košice, Slovakia
}

\begin{abstract}
Influence of the directional ordering on magnetization processes in the amorphous $\mathrm{Fe}_{44.5} \mathrm{Co}_{44.5} \mathrm{Zr}_{7} \mathrm{~B}_{4}$ alloy was investigated in the temperature region of 27 to $300^{\circ} \mathrm{C}$ for stabilization times of up to $1000 \mathrm{~min}$ by means of the Perminvar effect. The time-temperature dependent Perminvar effect was found to be very sensitive on the stage of the domain structure stabilization. The Perminvar critical fields $H_{\mathrm{CR}}$ were shifted to higher values with increasing stabilization temperatures and times. At lower temperatures, the susceptibility increases over wide range of the measuring fields. Stabilization at higher temperatures causes that the susceptibility abruptly increases in the narrow field range. The record critical field $H_{\mathrm{CR}}=246 \mathrm{Am}^{-1}$ was obtained.
\end{abstract}

PACS numbers: 75.50.Kj, 75.60.Lr

\section{Introduction}

Multicomponent $\mathrm{Fe}_{44.5} \mathrm{Co}_{44.5} \mathrm{Zr}_{7} \mathrm{~B}_{4}$ Hitperm alloy was prepared for high temperature magnetic applications [1]. Chemical composition, amorphous structure and ferromagnetic state bring about intensive time-temperature directional ordering in the alloy [2] stabilizing the actual domain structure and deepening the domain wall (DW) potentials manifested by several macroscopic phenomena [3]. One of them is the Perminvar effect (PE) characterized by constant permeability (susceptibility) for measuring field amplitudes $H_{0}<H_{\mathrm{CR}}$ [4]. Atom pairs reordering leads to formation of the additional stabilization DWs' potential $E_{\mathrm{s}}(t, T)[5]$ which influences the pinning field distribution. The critical field $H_{\mathrm{CR}}$ is the lowest pinning field. At this field the susceptibility stops to be constant and begins to rise [2]. The total relaxation consists of several elementary relaxation processes 
which contribute to the DW potential. The critical field is given by the gradient of the total DW potential [3]:

$$
H_{\mathrm{CR}}(T, t)=\left.\frac{1}{2 S I_{\mathrm{S}} \cos \phi_{0}} \frac{\mathrm{d} E(u, T, t)}{\mathrm{d} u}\right|_{\max }=H_{\mathrm{CR}_{0}}+\sum_{p=1}^{P} \Delta H_{\mathrm{CR}_{0}}^{(p)} R^{(p)}(T, t),(1)
$$

where $I_{\mathrm{s}}$ is the saturation magnetization, $H_{\mathrm{CR}_{0}}$ is the unrelaxed critical field, $P$ is the total number of the atom pair types, $\Delta H_{\mathrm{CR}_{0}}^{(p)}$ is a contribution to the maximal change of the critical field caused by $p$-type magnetic relaxation, $R^{(p)}(T, t)$ is the $p$-type relaxation function.

\section{Experimental}

Perminvar effect have been investigated on the amorphous $\mathrm{Fe}_{44.5} \mathrm{Co}_{44.5} \mathrm{Zr}_{7} \mathrm{~B}_{4}$ alloy by the AC susceptibility measurements. The amorphous alloy was prepared by the melt-spinning technique in ribbon form. The susceptibility measurements were carried out on the straight samples $5 \mathrm{~cm}$ long and $2 \mathrm{~mm}$ wide at temperatures $27-300^{\circ} \mathrm{C}$ after stabilization up to $1000 \mathrm{~min}$ in argon atmosphere. After each temperature the sample was cooled in the furnace and the Perminvar effect measurements were repeated at room temperature again. The field dependences of the AC susceptibility after demagnetization and stabilization were measured by the mutual induction method at frequency $f=334 \mathrm{~Hz}$.

\section{Results and discussion}

The influence of time-temperature stabilization of domain structure on magnetic processes in amorphous alloys can be investigated by the Perminvar effect.
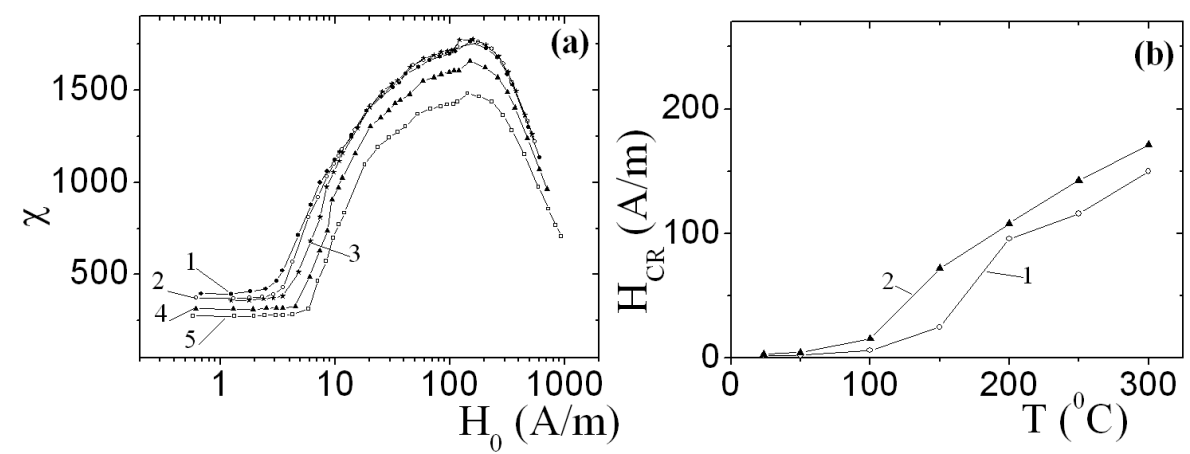

Fig. 1. (a) Dependence of the AC susceptibility $\chi$ on the magnetic field amplitude $H_{0}$ measured at temperature $T=50^{\circ} \mathrm{C}$ for stabilization times: $1-t_{\mathrm{s}}=1 \mathrm{~min}, 2-4 \mathrm{~min}$, $3-10 \mathrm{~min}, 4-40 \mathrm{~min}, 5-100 \mathrm{~min}$. (b) Temperature dependence of the Perminvar critical field measured for stabilization time curve: $1-1 \mathrm{~min}, 2-100 \mathrm{~min}$.

Figure 1a shows the amplitude dependences $\chi\left(H_{0}\right)$ measured at temperature $T=50^{\circ} \mathrm{C}$, after different times of stabilization $\left(t_{\mathrm{s}}\right)$. We can see there three main 
types of magnetization processes. At low magnetic fields, $H_{0}<H_{\mathrm{CR}}$, reversible movements of DWs are under way and susceptibility is constant. Increasing the time of stabilization the value of $H_{\mathrm{CR}}$ increases from $2 \mathrm{~A} / \mathrm{m}\left(t_{\mathrm{s}}=1 \mathrm{~min}\right)$ up to $4.6 \mathrm{~A} / \mathrm{m}\left(t_{\mathrm{s}}=100 \mathrm{~min}\right)$. At the same time $\chi$ decreases from 396 down to 276 . Exceeding $H_{\mathrm{CR}}$, the susceptibility is growing up abruptly. At the measuring fields $H_{0}>H_{\mathrm{CR}}$, irreversible movements of DWs take place. Continuous increase in $\chi\left(H_{0}\right)$ indicates a broad distribution of pinning fields $H_{\mathrm{p}}\left(H_{\mathrm{CR}}<H_{\mathrm{p}}<H_{\mathrm{max}}\right)$. For fields $H_{0}>H_{\max }$, the susceptibility begins to decrease and the magnetization of sample is carried out by a rotation of the magnetic moments. The Perminvar critical fields increase during stabilization temperature rising, too (Fig. 1b). The $H_{\mathrm{CR}}(T)$ were measured at two stabilization times $t_{\mathrm{s}}$. The higher values of $H_{\mathrm{CR}}$ belong to higher $t_{\mathrm{s}}$ at all temperatures. The critical fields for $t_{\mathrm{s}}=100 \mathrm{~min}$ monotonously increases from $H_{\mathrm{CR}}=3.2 \mathrm{~A} / \mathrm{m}$ at room temperature up to $H_{\mathrm{CR}}=$ $172 \mathrm{~A} / \mathrm{m}$ at $300^{\circ} \mathrm{C}$. It relates to growth of relaxation functions $R^{(p)}(T, t)(\mathrm{Eq} .(1))$ with rising temperature. The critical fields after $1 \mathrm{~min}$ stabilization reach lower values of $H_{\mathrm{CR}}$.
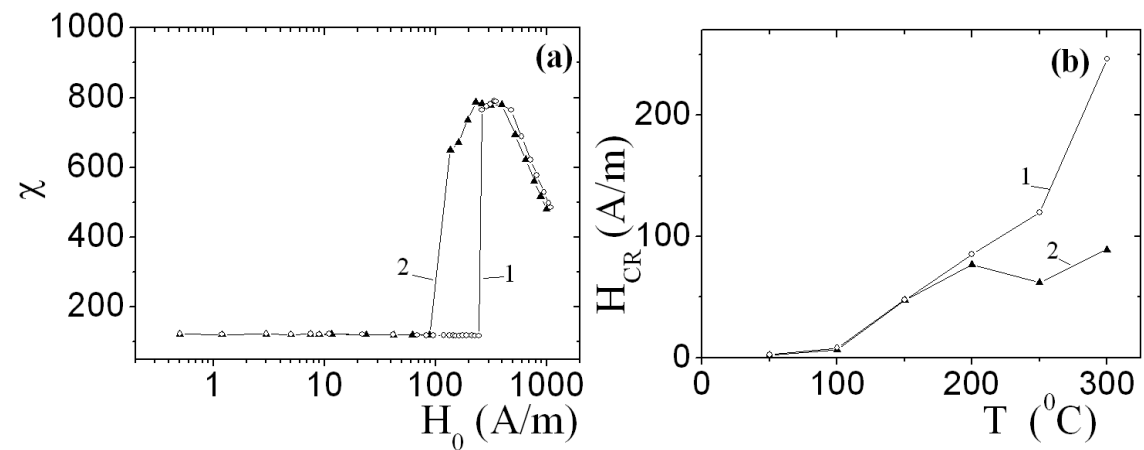

Fig. 2. (a) Dependence of the AC susceptibility $\chi$ on the magnetic field amplitude $H_{0}$ measured at temperature $T=27^{\circ} \mathrm{C}$ after 1000 min long cooling from $300^{\circ} \mathrm{C}$, curve 1 - without demagnetization, $2-t_{\mathrm{s}}=1 \mathrm{~min}$ after demagnetization. (b) Temperature dependence of the Perminvar critical field measured at room temperature after cooling from given temperatures (curves 1 and 2 - see Fig. 2a).

Studying the Perminvar effect in the amorphous Hitperm-type alloys, a conservation of perminvar properties for material cooling down to room temperature represents a great interest. In Fig. 2a one can see that the susceptibility $\chi\left(H_{0}\right)$ remains constant up to the value $H_{\mathrm{CR}}=246 \mathrm{~A} / \mathrm{m}$ after cooling from $T=300^{\circ} \mathrm{C}$ (curve 1 ). The susceptibility $\chi\left(H_{0}\right)$ abruptly increases almost to its maximal value if this $H_{\mathrm{CR}}$ is overrun. This giant $\chi\left(H_{0}\right)$ growth is connected with advanced development and simultaneous unification of the stabilization potential $E_{\mathrm{S}}(t, T)$ for all DWs and with a shift of the pinning field distribution to higher values. Consecutive demagnetization does not change this Perminvar character of the $\chi\left(H_{0}\right)$ depen- 
dences. The value of critical field $H_{\mathrm{CR}}$ obtained by cooling from $T=300^{\circ} \mathrm{C}$ to room temperature (RT) after 1 min long stabilization exceeds more than 40 times the $H_{\mathrm{CR}}$ value obtained for the as-cast sample after the same stabilization time (Fig. 1b, curve 1). It comes from the fact that DWs after demagnetization are placed to places where deep and abrupt potential wells were created at the previous intensive stabilization of the domain structure. The critical fields measured after cooling to RT monotonously increase with temperature of previous heatings (Fig. 2b, curve1). The record value of $H_{\mathrm{CR}}=246 \mathrm{~A} / \mathrm{m}$ was obtained after cooling from $300^{\circ} \mathrm{C}$. The conserved high values of critical fields are partly caused by irreversible relaxation processes at higher stabilization temperatures. After demagnetization and consecutive stabilization during $1 \mathrm{~min}$, the critical fields are lower than the $H_{\mathrm{CR}}$ obtained without demagnetization but they are still higher than the intensity of the earth magnetic field. This fact could be important for electronic applications requiring a constant value of the susceptibility in a wide range of magnetic field intensities.

\section{Conclusion}

In this work we presented that the intensive Perminvar effect occurs in the FeCoZrB Hitperm-type amorphous alloy. It is sensitive on time-temperature stabilization of the domain walls which is caused by the atom pairs directional ordering. The Perminvar critical fields increase with increasing time and temperature and they reach the record value of $H_{\mathrm{CR}}=246 \mathrm{~A} / \mathrm{m}$ after stabilization at $T=300^{\circ} \mathrm{C}$ and consecutive cooling to room temperature. The obtained Perminvar properties of the Hitperm-type alloy maintain for room temperature as well.

\section{Acknowledgments}

This work was supported by the VEGA grant no. 1/3035/06. M.E. McHenry is acknowledged for his kind supplying us by samples.

\section{References}

[1] M.E. McHenry, M.A. Willard, D.E. Laughlin, Prog. Mater. Sci. 44, 291 (1999).

[2] R. Andrejco, P. Vojtanik, J. Phys., Condens. Matter 16, 3745 (2004).

[3] P. Vojtanik, J. Magn. Magn. Mater. 304, 159 (2006).

[4] G.W. Elmen, J. Franklin Inst. 207, 583 (1929).

[5] L. Néel, J. Phys. Radium 13, 249 (1952). 